\title{
Association of monocyte to high-density lipoprotein cholesterol ratio with carotid artery intima-media thickness in patients with systemic lupus erythematosus
}

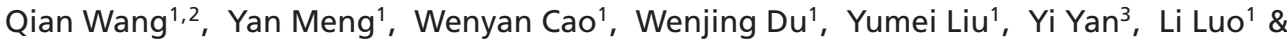 \\ Xiumin Ma*,1,3 \\ ${ }^{1}$ State Key Laboratory Incubation Base of Xinjiang Major Diseases Research, The First Affiliated Hospital of Xinjiang Medical \\ University, Urumqi, 830011, PR China \\ ${ }^{2}$ Clinical Laboratory Center, People's Hospital of Xinjiang Uygur Autonomous Region, Urumqi, 830001, PR China \\ ${ }^{3}$ Department of Basic Medicine, Xinjiang Medical University, Urumqi, 830011, PR China \\ * Author for correspondence: maxiumin1210@sohu.com
}

\begin{abstract}
Aim: The purpose of this study was to evaluate the relationship between monocyte to high-density lipoprotein cholesterol ratio (MHR) and carotid atherosclerostic plaque in patients with systemic lupus erythematosus (SLE). Methods: A total of 214 SLE patients were divided into two groups according to the results of ultrasonic examination: carotid arterial atherosclerotic plaque groups and noncarotid arterial atherosclerosis groups. Results: The values of monocyte to high-density lipoprotein-cholesterol ratio (MHR) increase in carotid arterial atherosclerotic plaque groups compared with noncarotid arterial atherosclerosis groups $(0.32 \pm 0.18$ vs $0.26 \pm 0.15 ; p=0.015)$. There was a significant correlation between MHR and carotid artery intima-media thickness $(r=0.228 ; p=0.001)$ in patients with SLE. Conclusion: Our study suggests that the values of MHR could be a marker to assess carotid artery intima-media thickness in patients with SLE.
\end{abstract}

First draft submitted: 11 December 2016; Accepted for publication: 30 October 2017; Published online: 23 March 2018

Keywords: carotid artery intima-media thickness $\bullet$ hypertension $\bullet$ inflammation $\bullet$ monocyte to high-density lipoprotein cholesterol ratio $\bullet$ oxidative stress $\bullet$ systemic lupus erythematosus

Systemic lupus erythematosus (SLE) is a chronic autoimmune disease that involves with the multiple organizations and organs. Investigator [1] reported that the incidence of cardiovascular diseases in SLE patients was approximately 7-8 times than that in general population. It is well accepted evidence that traditional cardiovascular risk factors may contribute to atherosclerosis in patients with SLE. Importantly, Sherer et al. [2] reported that atherosclerosis was found to be an initiator for cardiovascular disease in patients with SLE. Atherosclerotic plaque has been considered to be the main risk factor for coronary artery disease, and is a predictor for the occurrence of coronary artery disease [3]. Obviously, the examination for atherosclerotic plaque is crucial to estimate and prevent cardiovascular events in patients with SLE. Therefore, it is important to investigate an objective, convenient and reliable laboratory marker to assess carotid atherosclerostic plaque in clinical laboratory.

Monocyte to high-density lipoprotein cholesterol ratio (MHR) is closely related to inflammation and oxidative stress, which can be used as a prognostic indicator in patients with cardiovascular disease [4,5]. Kanbay et al. [6] demonstrated that increased values of MHR were associated with cardiovascular disease in patients with chronic kidney disease. There is a relationship between MHR and prognostic outcome in patients with coronary artery [7], and higher values of MHR can predict nephropathy development in patients with myocardial infarction after primary percutaneous transluminal coronary intervention. Similarly, Cetin et al. [8] suggested that MHR was a reliable marker in reflecting severity of coronary artery disease and predicting cardiovascular events in patients with acute coronary syndrome. In fact, inflammation and oxidative stress may contribute to explain the associations between elevated MHR and these diseases. However, inflammation and oxidative stress have been involved with 
carotid atherosclerostic in SLE patients. Hence, the purpose of this study was to evaluate the association of MHR with carotid atherosclerostic plaque in patients with SLE.

\section{Patients \& methods}

A total of 214 patients with SLE were recruited from First Affiliated Hospital of Xinjiang Medical University (Urumqi, China), which met the 1997 American College of Rheumatology (ACR) revised classification criteria. The key point to be examined in this paper: carotid IMT is a thickness that measured from the media-adventitia interface to the intima-lumen interface. Plaque is defined as a focal structure that encroaches into the arterial lumen of at least $0.5 \mathrm{~mm}$ or $50 \%$ of the surrounding IMT value or demonstrates a thickness of $>$ or $=1.5 \mathrm{~mm}$ as measured from the media-adventitia interface to the intima-lumen interface. In our study, all of our patients were examined by ultrasonography within 2 days after admission, so the laboratory data have certain reliability. Carotid artery intima-media thickness (cIMT) was discerned by ultrasound in all patients with SLE. We used LOGIQ9 color Doppler ultrasound diagnostic apparatus (probe frequency 7-10 MHz) to explore the carotid artery of both sides of the patient, all the patients were examined by three observers including the director of the department, all were blind, and there was no significant difference between the three groups, and recorded the size, number, shape and location - among others. The presence of carotid atherosclerotic plaques was defined with intima-media thickness greater than $1.5 \mathrm{~mm}[9,10]$. According to the traditional risk assessment, clinical symptom and correlation detection, the patients with following diseases or conditions were excluded: cardiovascular disease, hypertension, diabetes, infectious disease, cancer, smoking, anemia and other autoimmune diseases. In addition, one of the exclusion criteria in this study is anemia, the range of anemia we used: adult male $\mathrm{Hb}<120 \mathrm{~g} / \mathrm{l}$, adult female (nonpregnant) $\mathrm{Hb}<110 \mathrm{~g} / \mathrm{l}$. It is really true that our retrospective design is a study limitation.

In this study, we retrospectively extracted clinical and laboratory data of all SLE patients. The blood routine tests were evaluated by using the Sysmex XN-1000 automatic blood instrument. The biochemical examinations were measured by using automatic biochemical analyzer. This clinical research complies with Helsinki Declaration, and this study has been approved by the Institutional Review Board in First Affiliated Hospital of Xinjiang Medical University, and obtained the informed consents of all patients.

\section{Statistical analysis}

The data were analyzed with the SPSS 16.0 statistical software (SPSS Inc., IL, USA). Continuous variables were expressed as mean \pm standard deviation. K-S test was used to compare data with normal distribution. The Student's t-test, Mann-Whitney U test and $\chi^{2}$ test were used to compare the differences between the two groups as appropriate. The correlations between the laboratory parameters were analyzed by using Spearman correlation. We used logistic multiple regression analysis to analyze laboratory marker associated with carotid atherosclerotic plaques. Receiver operating characteristic curve analysis was used to analyze MHR in assessing the atherosclerosis in patients with SLE. $\mathrm{p}<0.05$ was considered statistically significant.

\section{Results}

A total of 214 SLE patients were divided into two groups according to the results of ultrasonic examination: carotid arterial atherosclerotic plaque (CAP) groups and noncarotid arterial atherosclerosis (NCAP) groups. The clinical and laboratory data from the two groups are shown in Table 1. There were statistical differences in total cholesterol (TC), C-reactive protein (CRP), hemoglobin, lymphocyte counts, monocyte counts (MC), neutrophil counts (NC), triglyceride (TG) and total protein between the two groups. Of note, the values of MHR increased in CAP groups compared with NCAP groups $(0.32 \pm 0.18$ vs $0.26 \pm 0.15 ; \mathrm{p}=0.015)$.

The correlation analysis between MHR and other parameters were carried out by Spearman correlation. The results showed that increased MHR was positively correlated with CRP, NC, MC and TG $(r=0.506, p=0.016$; $\mathrm{r}=0.555, \mathrm{p}=0.007 ; \mathrm{r}=0.602, \mathrm{p}=0.003 ; \mathrm{r}=0.474, \mathrm{p}=0.022)$, and negatively correlated with high-density lipoprotein cholestrol (HDL-C) and hemoglobin $(r=-0.533, p=0.008 ; r=-0.259, p=0.041)$ in all SLE patients. There was a significant correlation between MHR and $\operatorname{cIMT}(r=0.228, p=0.001)$ in patients with SLE (Figure 1).

Multiple logistic regression analysis showed that increased MHR values were independently related with CAP (odds ratio: 1.011; 95\% CI: 1.004-1.018; $\mathrm{p}=0.002$ ) in patients with SLE, when gender, age, BMI, TC, TG, low-density lipoprotein cholesterol, CRP, fasting blood glucose, hemoglobin, NC, total protein, mediation use and MHR were included as independent variables, as shown in Table 2. The predictive value of MHR was assessed by receiver operating characteristic curve, indicating that the area under the curve of MHR in determining CAP in 
Table 1. Clinical and laboratory characteristics in carotid arterial atherosclerotic plaque groups and noncarotid arterial atherosclerosis groups in systemic lupus erythematosus patients.

\begin{tabular}{|c|c|c|c|}
\hline Clinical laboratory index & $\begin{array}{l}\text { CAP groups } \\
n=105\end{array}$ & $\begin{array}{l}\text { NCAP groups } \\
n=109\end{array}$ & p-value \\
\hline Gender (female) & $89(84.8 \%)$ & $97(89.6 \%)$ & 0.359 \\
\hline Age (year) & $47.2 \pm 7.06$ & $42.2 \pm 6.29$ & 0.502 \\
\hline BMI $\left(\mathrm{kg} / \mathrm{m}^{2}\right)$ & $27.2 \pm 3.54$ & $26.5 \pm 3.18$ & 0.624 \\
\hline Disease duration (year) & $11.8 \pm 5.01$ & $6.6 \pm 1.96$ & 0.005 \\
\hline \multicolumn{4}{|l|}{ Mediation use $(n, \%)$} \\
\hline Glucocorticoid & $12(9.6 \%)$ & $16(8.6 \%)$ & 0.752 \\
\hline Azathioprine & $10(8.0 \%)$ & $14(7.5 \%)$ & 0.868 \\
\hline Cyclophosphamide & $7(5.6 \%)$ & $11(5.9 \%)$ & 0.917 \\
\hline Nonsteroidal anti-inflammatory drugs & $87(69.6 \%)$ & $113(60.4 \%)$ & 0.098 \\
\hline Lymphocyte counts $\left(10^{9} / \mathrm{l}\right)$ & $1.1 \pm 0.37$ & $1.7 \pm 0.62$ & 0.026 \\
\hline Neutrophil counts $\left(10^{9} / \mathrm{I}\right)$ & $3.9 \pm 2.03$ & $2.3 \pm 0.59$ & 0.024 \\
\hline Hemoglobin $\left(10^{9} / \mathrm{l}\right)$ & $102.8 \pm 17.32$ & $120.9 \pm 16.41$ & 0.021 \\
\hline Monocyte counts $\left(10^{9} / \mathrm{l}\right)$ & $0.5 \pm 0.21$ & $0.4 \pm 0.16$ & 0.013 \\
\hline Platelet counts $\left(10^{9} / \mathrm{l}\right)$ & $178.3 \pm 97.95$ & $159.6 \pm 85.86$ & 0.639 \\
\hline C-reactive protein (mg/l) & $10.9 \pm 6.71$ & $2.2 \pm 0.86$ & 0.001 \\
\hline Erythrocyte sedimentation rate $(\mathrm{mm} / \mathrm{h})$ & $50.0 \pm 16.22$ & $45.9 \pm 23.63$ & 0.646 \\
\hline Alanine transaminase $(\mathrm{U} / \mathrm{I})$ & $23.5 \pm 6.88$ & $25.8 \pm 4.35$ & 0.361 \\
\hline Aspartate aminotransferase $(\mathrm{U} / \mathrm{I})$ & $24.9 \pm 7.42$ & $33.3 \pm 6.51$ & 0.284 \\
\hline Creatinine $(\mu \mathrm{mol} / \mathrm{l})$ & $65.3 \pm 21.45$ & $53.8 \pm 23.02$ & 0.238 \\
\hline Total protein $(\mathrm{g} / \mathrm{l})$ & $66.8 \pm 8.76$ & $59.4 \pm 6.89$ & 0.041 \\
\hline $\begin{array}{l}\text { Low-density lipoprotein cholesterol } \\
(\mathrm{mmol} / \mathrm{l})\end{array}$ & $2.3 \pm 0.54$ & $2.5 \pm 1.10$ & 0.622 \\
\hline $\begin{array}{l}\text { High-density lipoprotein cholesterol } \\
(\mathrm{mmol} / \mathrm{l})\end{array}$ & $1.5 \pm 0.49$ & $1.7 \pm 0.63$ & 0.057 \\
\hline Total cholesterol (mmol/l) & $4.5 \pm 0.83$ & $3.3 \pm 1.25$ & 0.010 \\
\hline Triglyceride (mmol/l) & $2.9 \pm 1.19$ & $1.8 \pm 0.69$ & 0.012 \\
\hline Fasting blood glucose (mmol/l) & $4.9 \pm 0.77$ & $5.7 \pm 2.05$ & 0.251 \\
\hline MHR & $0.32 \pm 0.18$ & $0.26 \pm 0.15$ & 0.015 \\
\hline
\end{tabular}

Table 2. Multivariate logistic regression analysis showing the independent factors associated with carotid arterial atherosclerotic plaque in systemic lupus erythematosus patients.

\begin{tabular}{|lllr}
\hline Comparison of a variety of risk factors & OR & p-value & $95 \%$ Cl \\
\hline Age & 1.033 & 0.028 & $1.007-1.125$ \\
\hline C-reactive protein & 1.041 & 0.013 & $1.022-1.060$ \\
\hline Disease duration & 1.368 & $<0.001$ & $1.211-1.545$ \\
\hline Total cholesterol & 1.096 & 0.012 & $1.016-1.211$ \\
\hline Triglyceride & 1.008 & 0.036 & $1.001-1.015$ \\
\hline MHR & 1.011 & 0.002 & $1.004-1.018$ \\
\hline
\end{tabular}

MHR: Monocyte to high-density lipoprotein cholesterol ratio; OR: Odds ratio.

SLE patients was 0.842 (CI\%: $0.754-0.902 ; \mathrm{p}=0.007$ ) with the sensitivity of 0.833 and the specificity of 0.800 , respectively, and the cut-off value was 0.39 for MHR in predicting CAP in patients with SLE.

\section{Discussion}

Our study found that the values of MHR were significantly higher in SLE patients with carotid atherosclerotic plaques than those without carotid atherosclerotic plaques, and increased MHR was related to SLE patients with carotid atherosclerotic plaques in logistic regression analysis. Interestingly, the values of MHR were positively 


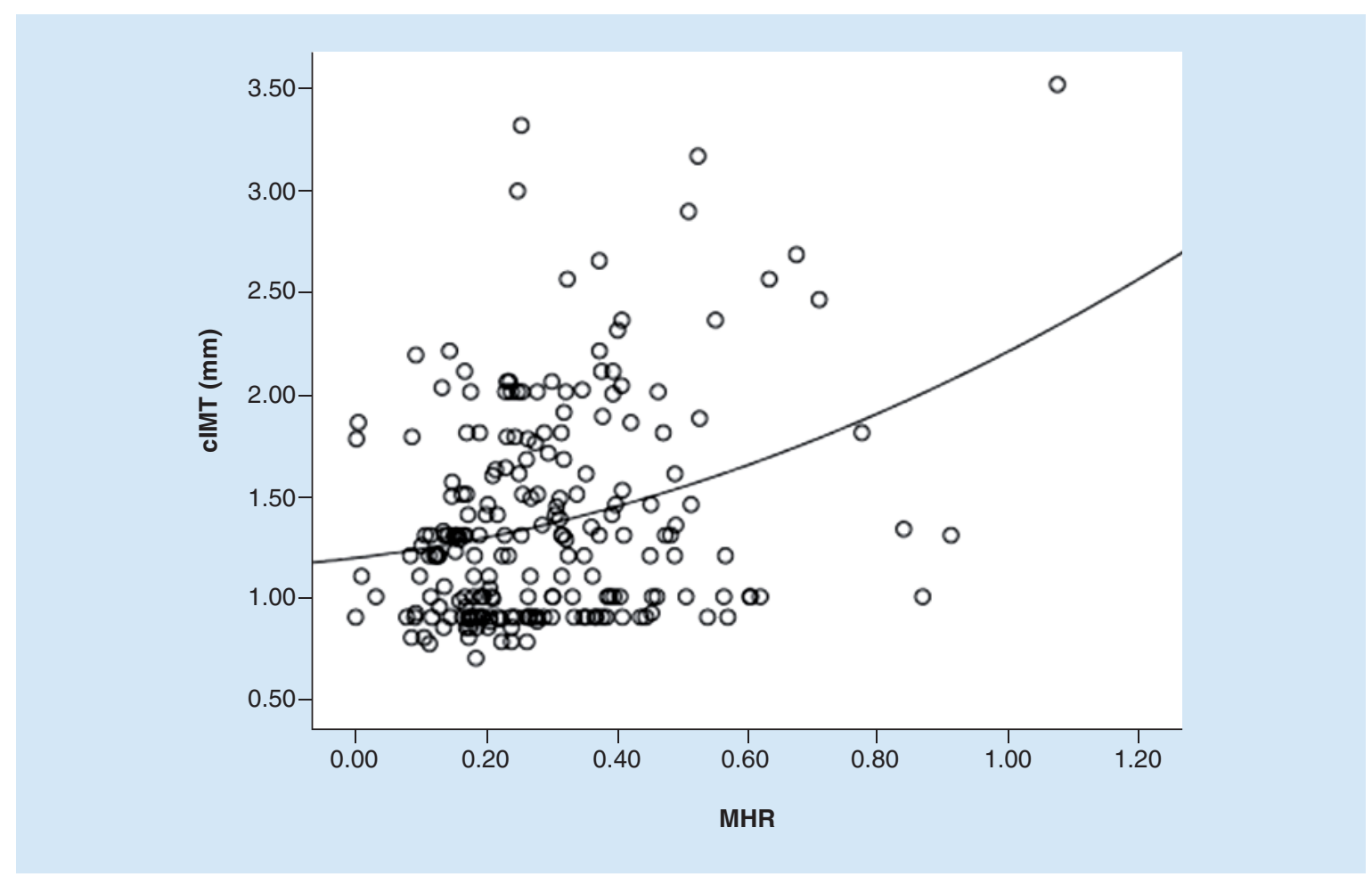

Figure 1. A significant scatter diagram between monocyte to high-density lipoprotein cholesterol ratio and carotid artery intima-media thickness in patients with systemic lupus erythematosus.

CIMT: Carotid artery intima-media thickness; MHR: Monocyte to high-density lipoprotein cholesterol ratio.

correlated with cIMT in SLE patients. But it is weak and the relation of other traditional risk factors (age, CRP and TC ) are stronger. We also found the statistical difference in disease duration between both groups of patients. Our analysis may be due to clinical symptoms, the patient in NCAP group may not have obvious symptoms without timely medical diagnosis, and the CAP group of patients may have an early complication, which can be diagnosed early. MHR has been considered as an inflammatory marker in various diseases. More and more evidences indicated that inflammatory process play an important role in development of atherosclerosis, and some inflammatory cytokines were related to atherosclerosis in patients with SLE [11]. Our study observed that increased MHR are positively correlated with cIMT in SLE patients. Cardiac and cerebral vascular diseases associated with atherosclerosis are severe complications in patients with SLE. Ultrasonic examinations were significantly affected by anatomical variations and subjective factors in establishing the diagnosis of carotid atherosclerotic plaques. Compared with CRP, ESR and ultrasonic examinations, MHR is an objective measurement in blood routine tests with no additional cost. The possible biological mechanism for the observed association of MHR with cIMT is not well known in patients with SLE, inflammatory and oxidative stress may be an intermediate medium to link the relationship between MHR and cIMT in SLE patients. Recent study also found that monocyte cell counts can predict the occurrence of macrovascular complications in patients with diabetes mellitus [12]. In fact, immune factors may induce and activate monocytes in vitro, which produces some inflammatory cytokines such as IL-6, IL-1 and TNF to release in the body [13,14]. Monocytes are involved with the pathogenesis of some diseases, abnormal activation can generate acute and chronic inflammation and monocytes are the most pivotal cell for secretion of proinflammatory and pro-oxidant cytokines at the site of inflammation [14]. Monocytes are activated by chronic inflammation in chronic kidney disease patients [6]. There is evidence that MC increased in patients with myocardial infarction, since acute inflammation stimulate monocytes from bone marrow into peripheral circulation [15]. These results may lead to increase in MC in patients with SLE. Previous studies have shown or suggested that SLE can affect all three blood cell lines and include anemia, leukopenia and thrombocytopenia. Leukopenia is very common in SLE patients, occurring in approximately $50 \%$ of patients. According to research reports, leukopenia has the following main reasons: autoantibodies mediate the destruction of hematopoiesis precursor cell and mature 
cells; TNF-mediated cell apoptosis; and drug intervention. However, granulocytes and lymphocytes were largely reduced; monocytes were not significantly affected [16-18]. Knowing that lower HDL-C has been considered to be a risk factor in general population. To our knowledge, HDL-C is an independent protective factor in cardiovascular disease. In addition, HDL-C has anti-inflammatory, antioxidant and antithrombotic properties, even in general population, and chronic inflammation has impact on the normal physiological metabolism of HDL [19,20]. It has been demonstrated that lower concentrations of HDL were negatively associated with increased inflammatory biomarkers in plasma [21]. On the contrary, inflammatory response causes the change of function and composition of HDL-C [22,23]. The information may provide a possible fact that inflammatory progress tends to decrease HDL-C in patients with SLE, the conditions are more evident in SLE patients with carotid atherosclerotic plaques. Therefore, elevated MHR may be attributed to the increased MC and decreased HDL-C in SLE patients with carotid atherosclerotic plaques.

The first limitation of this study was a small sample size in this cross-sectional study. Second, long-term immunosuppressive medications use might influence blood cell components in patients with SLE, although medication use was considered as confounders in logistic multiple regression analysis. Third, this study has not investigated whether anti-inflammatory therapy had an influence for MHR values in patients with SLE. Nevertheless, our study suggests that MHR is associated with SLE patients with carotid atherosclerotic plaques and MHR is positively correlated with cIMT in SLE patients. The values of MHR may be a marker to assess cIMT in patients with SLE.

\section{Summary points}

- The values of monocyte to high-density lipoprotein cholesterol ratio (MHR) were significantly higher in systemic lupus erythematosus (SLE) patients with carotid atherosclerotic plaques than those without carotid atherosclerotic plaques.

- The values of MHR were positively correlated with carotid artery intima-media thickness in SLE patients.

- The size of the atherosclerotic plaque in patients with SLE is associated with MHR.

- MHR is an objective measurement in blood routine tests with no additional cost for diagnosing SLE.

Financial \& competing interests disclosure

The work was supported by a grant from National Natural Science Foundation of China (81760372). The authors have no other relevant affiliations or financial involvement with any organization or entity with a financial interest in or financial conflict with the subject matter or materials discussed in the manuscript apart from those disclosed.

No writing assistance was utilized in the production of this manuscript.

\section{Ethical conduct of research}

The authors state that they have obtained appropriate institutional review board approval or have followed the principles outlined in the Declaration of Helsinki for all human or animal experimental investigations. In addition, for investigations involving human subjects, informed consent has been obtained from the participants involved.

\section{Open access}

This work is licensed under the Attribution-NonCommercial-NoDerivatives 4.0 Unported License. To view a copy of this license, visit http://creativecommons.org/licenses/by-nc-nd/4.0/

\section{References}

Papers of special note have been highlighted as: $\bullet$ of interest; $\bullet \bullet$ of considerable interest

1 Manzi S, Meilahn EN, Rairie JE et al. Age-specific incidence rates of myocardial infarction and angina in woman with systemic lupus erythematosus: comparison with the Framingham Study. Am. J. Epidemiol. 145(5), 408-415 (1997).

2 Sherer Y, Zinger H, Shoenfeld Y. Atherosclerosis in systemic lupus erythematosus. Autoimmunity 43(1), 98-102 (2010).

3 Haixia Yang. Study on the correlation between thickness of carotid artery and femoral artery intima-media and coronary artery disease. Hebei Med. J. 30(7), 960-962 (2008).

4 Canpolar U, Cetin EH, Cetin S et al. Association of monocyte-to-HDL cholesterol ratio with slow coronary flow is linked to systemic inflammation. Clin. Appl. Thromb. Hemost. 22(5), 476-482 (2016). 
- Suggests that higher monocyte to high-density lipoprotein cholesterol ratio (MHR) which indicates an enhanced inflammation and oxidative stress was significantly and independently associated with the presence of slow coronary flow. Besides, MHR was positively correlated with serum high-sensitivity C-reactive protein (hsCRP) level as a conventional marker for systemic inflammation.

5 Kundi H, Gok M, Kiziltunc E et al. Relation between monocyte to high-density lipoprotein cholesterol ratio with presence and severity of isolated coronary artery ectasia. Am. J. Cardiol. 116(11), 1685-1689 (2015).

- Investigates an easily available inflammatory and oxidative stress marker and proved MHR cholesterol ratio is associated with the severity of coronary artery ectasia.

6 Kanbay M, Solak Y, Unal HU et al. Monocyte count/HDL cholesterol ratio and cardiovascular events in patients with chronic kidney disease. Int. Urol. Nephrol. 46(8), 1619-1625 (2014).

-• Reports for the first time that an increased $\mathrm{M} / \mathrm{H}$ ratio was cross-sectionally associated with a worse cardiovascular profile and arose as independent predictors of major cardiovascular events during follow-up.

7 Akboga MK, Balci KG, Maden O et al. Usefulness of monocyte to HDL-cholesterol ratio to predict high SYNTAX score in patients with stable coronary artery disease. Biomark. Med. 10(4), 375-383 (2016).

8 Cetin MS, Ozcan Cetin EH, Kalender E et al. Monocyte to HDL cholesterol ratio predicts coronary artery disease severity and future major cardiovascular adverse events in acute coronary syndrome. Heart Lung Circ. 25(11), 1077-1086 (2016).

9 Touboul PJ, Hennerici MG, Meairs S et al. Mannheim intima-media thickness consensus. Cerebrovasc. Dis. 18(4), 346-349 (2004).

10 Borhani NO, Mercuri M, Borhani PA et al. Final outcome results of the Multicenter Isradipine Diuretic Atherosclerosis Study (MIDAS). a randomized controlled trial. JAMA 276(10), 785-791 (1996).

11 Shovman O, Gilburd B, Shoenfeld Y. The role of inflammatory cytokines in the pathogenesis of systemic lupus erythematosus-relatedatherosclerosis: a novel target for treatment? J. Rheumatol. 33(3), 445-447 (2006).

12 Matsumura T, Taketa K, Motoshima $\mathrm{H}$ et al. Association between circulating leukocyte subtype counts and carotid intima-media thickness in Japanese subjects with Type 2 diabetes. Cardiovasc. Diabetol. 12, 177 (2013).

- Investigated the correlation between leukocyte subtype counts and intima-media thickness of the common carotid artery in subjects with Type 2 diabetes.

13 Wenping Sun. The Activating Effects And Potential Mechanisms Of Immune Complexes On Human Endothelial Cells And Monocytes [PhD thesis]. Shan Dong University, China (2013).

14 Ancuta P, Wang J, Gabuzda D. CD16 monocytes produce IL-6, CCL-2 and matrix metalloproteinase-9 upon interaction with CX3CL1-expressing endothelial cells. J. Leukoc. Biol. 80, 1156-1164 (2006).

- Suggests that immune factors may induce and activate monocytes in vitro, which produces some inflammatory cytokines such as IL-6, IL-1 and TNF to release in the body.

15 Dutta P, Nahrendorf M. Monocytes in myocardial infarction. Arterioscler. Thromb. Vasc. Biol. 35(5), 1066-1070 (2015).

16 Font J, Cervera R, Ramos-Casals $\mathrm{M}$ et al. Clasters of clinical and features in systemic lupus erythmatosus: analysis of 600 patients from a single center. Semin. Arthritis Rheum. 33(4), 217-230 (2004).

- Summarizes recent research into the mechanisms as to how elevated levels of triglyceride and low levels of high-density lipoprotein cholesterol contribute to inflammation and atherosclerosis.

17 Matsuyama W, Yamamoto M, Higashimoto I et al. TNF-related apoptosis-inducing ligand is involved in neutropenia of systemic lupus erythmatosus. Blood 104(1), 184-191 (2004).

18 Chen M, Zhao MH, Zhang Y et al. Antineutrophil autoantibodies and their target antigens in systemic lupus erythmatosus. Lupus 13(8), 584-589 (2004).

- The report proved that apoB level correlated with hsCRP level but was not the major determinant of hsCRP.

19 Welty FK. How do elevated triglycerides and low hdl-cholesterol affect inflammtion and atherothrombosis? Curr. Cardiol. Rep. 15(9), 400 (2013).

- Identified new markers of potential relevance to the pathways linked to high-density lipoprotein dysfunction in chronic hemodialysis.

20 Goswami B, Rajappa M, Singh B et al. Inflammation and dyslipidaemia: a possible interplay between established risk factors in north Indian males with coronary artery disease. Cardiovasc. J. Afr. 21(2), 103-108 (2010).

$21 \mathrm{Xu} \mathrm{W}$, Li R, Zhang $\mathrm{S}$ et al. The relationship between high-sensitivity C-reactive protein and apob, apob/apoal ratio in general population of China. Endocrine 42(1), 132-138 (2012).

22 Mange A, Goux A, Badiou $S$ et al. Hdl proteome in hemodialysis patients: a quantitative nanoflow liquid chromatography-tandem mass spectrometry approach. PLoS ONE 7(3), e34107 (2012).

23 Singh HV, Shrivastava AK, Raizada A et al. Atherogenic lipid profile and high sensitive C-reactive protein in patiens with rheumatoid arthritis. Clin. Biochem. 46(12), 1007-1012 (2013). 